\title{
Temperature jump and slip velocity calculations from an anisotropic scattering kernel
}

\author{
S. Kokou Dadzie* and J. Gilbert Méolans ${ }^{\dagger}$ \\ Université de Provence, UMR CNRS 6595 \\ Département de Mécanique Energétique - IUSTI \\ 5, rue Enrico Fermi 13453 Marseille cedex 13 France
}

(Dated: April 8, 2005)

This article deals with the problem of temperature jump and slip velocity at the wall in gas/surface interaction. A consistent modelling of an impermeable surface involving an anisotropic scattering kernel developed in previous works is used to establish boundary conditions in unstructured molecule gas flows. Thus a temperature jump relation is derived in which the gas viscous effects at the wall and the mean velocity gradients appear. Likewise, a slip velocity relation is obtained in which both the slip coefficient and the thermal creep coefficient depend on the wall-to-gas temperature ratio. Moreover, both the temperature jump and the slip velocity relations involve not only one accommodation coefficient as in usual expressions, but also the gas/surface information through the various (notably normal and tangential) accommodation coefficients of the momentum components.

PACS numbers: $51.10 .+\mathrm{y}$, 47.45.Gx, 47.27.Lx

Keywords: gas-wall interaction; Boltzmann equation; temperature jump; slip velocity; slip flows

\footnotetext{
*Electronic address: Kokou.Dadzie@polytech.univ-mrs.fr

${ }^{\dagger}$ Electronic address: Gilbert. Meolans@polytech.univ-mrs.fr
} 


\section{INTRODUCTION}

The validity domain of the continuum equations can be extended up to higher Knudsen numbers in the range corresponding to the slip regime, like in moderately rarefied gas flows and in microflows, if correct slip velocity and temperature jump relations are used as boundary conditions. In this regard, it is necessary, for modelling physical phenomena at the mean free path scale, to obtain accurate slip and jump conditions on the wall. So doing, a good form of boundary condition for the Boltzmann equation is also obtained.

Experiments in microflows (MEMS, microconduct) showed that, in this flow regime, pressure gradients, temperature gradients and velocity gradients become strongly sensitive and interlinked even at low speeds [1]. A well known phenomenon appearing under these conditions is the thermal creep [2-5]. In view of these effects, the macroscopic gradients interplay and the relevant phenomena, such as temperature or pressure variations due to the viscous dissipation or to the transformation of mechanical energy into internal energy, should receive consideration other than in ordinary flows.

In the usual formulation of the temperature jump problem, the heat conduction is considered in a rarefied gas bounded by a plane wall exposed to a uniform heat flow normal to the wall $[6-9]$. In this formulation, the temperature jump is governed by the temperature gradient only and the gas macroscopic motion is not taken into account. This treatment would be consistent with the negligible effects of the irreversible conversion of mechanical energy into internal energy, i.e heat energy, via the viscous dissipation in the flow. However, at the boundary, this argument is contradicted by the macroscopic gradient sensitivity and the surface effects [10-12]. Thus the gas rubbing against the surface can cause temperature variations at the wall. On the other hand, temperature jump relations are generally derived using either a complete diffuse scattering kernel $[7,13,14]$ or at best using only one accommodation coefficient in the surface modelling [8, 9, 15-17]. A very similar way is generally used to obtain the slip velocity $[2,18,19]$. However it is well known that the completely diffuse scattering kernel is not a good surface description [20]. Furthermore the 
single accommodation coefficient process is a rough qualitative description of the particle reflection at the wall related to the Maxwell boundary condition formulation[2]: Maxwell boundary condition exhibits only one accommodation coefficient for the accommodation of any kinetic properties whereas different accommodation coefficients can be calculated for each kinetic property (i.e. the three components of the momentum and energy)[21, 22]. Slip equations involving various accommodation coefficients have rarely been presented [23].

In this article, we consider more generally the problem of boundary conditions on the temperature and on the macroscopic velocity from a microscopic level description and using the complete Chapman-Enskog approximation for the distribution function of the incoming particles only. So doing, contrary to the standard formulation [6-9], the temperature jump problem is connected with the gas macroscopic motion at the wall and so the slip velocity problem is completely coupled with the temperature jump problem. The reflection law governing the reflection of the distribution function at the boundary is described by an anisotropic scattering kernel [24], where the gas/surface interaction information lies on the various accommodation coefficients of the momentum components. The result provides more general relations for the thermal boundary condition and for the slip velocity.

In section II the scattering kernel is briefly presented. In section III the problem of thermal boundary condition is investigated. The temperature jump so established appears as a more complete temperature jump relation depending on viscous effects, gas compressibility, and surface anisotropy. Section IV deals with the boundary condition on the gas mean velocity. Although the slip velocity is structured like the previous slip velocity formula including the thermal creep, it depends on the temperature jump and also involves the tangential gradients of the normal mean velocity at the wall. The theoretical values so predicted for the slip coefficient are compared to measures. 


\section{MODEL OF ANISOTROPIC SCATTERING KERNEL}

$\xi\left(\xi_{x}, \xi_{y}, \xi_{z}\right)$ denotes the velocity of reflected particles at the wall $\left(\xi \in\left\{\Omega=\mathbb{R}_{+} \times \mathbb{R} \times \mathbb{R}\right\}\right)$, $\xi^{\prime}\left(\xi_{x}^{\prime}, \xi_{y}^{\prime}, \xi_{z}^{\prime}\right)$ denotes the velocity of impinging particles $\left(\xi^{\prime} \in\left\{\Omega^{\prime}=\mathbb{R}_{-} \times \mathbb{R} \times \mathbb{R}\right\}\right)$ and $(x, y, z)$ are the three spatial coordinates with $x$ the normal axis to the wall oriented from the wall toward the gas. $U\left(U_{x}, U_{y}, U_{z}\right)$ is the gas macroscopic velocity. $V=\xi-U$ and $V^{\prime}=\xi^{\prime}-U$ are the peculiar velocities respectively for reflected and impinging particles. Since the normal component of the macroscopic velocity will be equal to zero at the wall, we will also have at the wall $(x=0), V \in \Omega$ and $V^{\prime} \in \Omega^{\prime} ; n$ and $T$ will respectively denote the gas numerical density and the gas temperature, and are obviously functions of $(x, y, z)$. The subscript "0" will denote the macroscopic parameters of the gas at the wall, but this subscript is omitted for the numerical density and for the gradients which are taken at the wall in any further result in the article.

The scattering kernel developed in reference [24] for the surface modelling may be written in a factorized form:

$$
\begin{array}{r}
B\left(\xi^{\prime}, \xi\right)=\left(\left(1-\alpha_{x}\right) \delta\left(\xi_{x}^{\prime}+\xi_{x}\right)+\alpha_{x} \frac{2 \xi_{x}}{C_{w}^{2}} e^{-\frac{\xi_{x}^{2}}{C_{w}^{2}}}\right)\left(\left(1-\alpha_{y}\right) \delta\left(\xi_{y}^{\prime}-\xi_{y}\right)+\right. \\
\left.\alpha_{y} \frac{1}{C_{w} \sqrt{\pi}} e^{-\frac{\xi_{y}^{2}}{C_{w}^{2}}}\right)\left(\left(1-\alpha_{z}\right) \delta\left(\xi_{z}^{\prime}-\xi_{z}\right)+\alpha_{z} \frac{1}{C_{w} \sqrt{\pi}} e^{-\frac{\xi_{z}^{2}}{C_{w}^{2}}}\right)
\end{array}
$$

where $C_{w}^{2}=\frac{2 k T_{w}}{m}$, with $T_{w}$ the wall temperature, $k$ the Boltzmann constant, and $m$ the molecular mass of the gaseous particles. Further on, we will note $C_{0}^{2}=\frac{2 k T_{0}}{m}$, with $T_{0}$ the temperature of the gas at $x=0$. Unlike in some previous models using unknown coefficients, the three coefficients involved in the scattering kernel (1), $\alpha_{x}, \alpha_{y}$, and $\alpha_{z}$, are shown to equal the three accommodation coefficients of the fluxes of the momentum components [24]. Thereby, these parameters cannot take an arbitrary value. Moreover, this model includes various kinds of accommodation processes, and allows distinguishing the accommodations of the different kinetic properties. 


\section{ON THE GAS TEMPERATURE AT $x=0$}

A correct microscopic level modelling at the boundary cannot be formulated using a single distribution function for the particles at the wall but using incident $f^{-}$and reflected $f^{+}$distribution functions. Accordingly, the boundary condition for the temperature can be derived from different accounts of the heat flux at the surface $x=0$. Practically, the heat flux obtained from the local expression using the two particle distribution functions $\left(f^{-}\right.$and $\left.f^{+}\right)$is equaled to the usual heat flux definition given by the whole particle conception.

Let us note $Q_{0}$ the gas heat flux per surface unit through the wall expressed at $x=0$, $\vec{Q}$ the heat flux vector at the same point, and $\vec{n}$ the normal vector oriented from the gas towards the wall, thus:

$$
\vec{Q} \cdot \vec{n}=Q_{0}
$$

Using the Fourier law, with the gas heat conductivity $\lambda_{c}$

$$
\vec{Q} \cdot \vec{n}=-\left(-\lambda_{c} \nabla T\right)=\lambda_{c} \frac{\partial T}{\partial x}
$$

on the other hand, from the kinetic theory formalism, $Q_{0}$ is expressed by

$$
Q_{0}=\int_{\Omega^{\prime}}\left[\frac{1}{2} m\left|V_{x}^{\prime}\right| V^{\prime 2} f^{-}\right] d_{\xi^{\prime}}-\int_{\Omega}\left[\frac{1}{2} m\left|V_{x}\right| V^{2} f^{+}\right] d_{\xi}
$$

The reflected and the incoming distribution functions are related at the wall as follows:

$$
\left|V_{x}\right| f^{+}=\int_{\Omega^{\prime}}\left|V_{x}^{\prime}\right| f^{-} B\left(\xi^{\prime}, \xi\right) d_{\xi^{\prime}}
$$

From relation (5) the local heat flux expression at the wall is written

$$
Q_{0}=\int_{\Omega^{\prime}}\left[\frac{1}{2} m\left|V_{x}^{\prime}\right| V^{\prime 2} f^{-}\right] d_{\xi^{\prime}}-\int_{\Omega^{\prime}}\left[\frac{1}{2} m\left|V_{x}^{\prime}\right| f^{-} \int_{\Omega} V^{2} B\left(\xi^{\prime}, \xi\right) d_{\xi}\right] d_{\xi^{\prime}} .
$$

In this last integral, the $\int_{\Omega} V^{2} B\left(\xi^{\prime}, \xi\right) d_{\xi}$ factor can be calculated separately using the scattering kernel (1) (see detailed calculation in Appendix). Then the local heat flux expression (6) becomes

$$
Q_{0}=\frac{1}{2} m \int_{\Omega^{\prime}}\left[\left(\alpha_{x}{V_{x}^{\prime 2}}^{2}+\alpha_{y} V_{y}^{\prime 2}+\alpha_{z} V_{z}^{\prime 2}\right)-\sigma_{0} C_{w}^{2}-\alpha_{y} U_{y}^{2}-\alpha_{z} U_{z}^{2}\right]\left|V_{x}^{\prime}\right| f^{-} d_{V^{\prime}}
$$


where

$$
\sigma_{0}=\left(\alpha_{x}+\frac{1}{2}\left(\alpha_{y}+\alpha_{z}\right)\right)
$$

Now we have to choose an incoming distribution function $f^{-}$. Previous approaches generally considered a simplified version of Chapman-Enskog distribution function where all velocity gradients were cancelled; furthermore in the various methods employed, the gas motion is rarely taken into account $[6-8,13]$. Considering the fact that the incoming particle distribution function should be close to the particle distribution function outside the Knudsen layer, precisely close to that used to derive the complete continuum equations, we consider here the full version of the Chapman-Enskog distribution including all the terms [9]. It is to be noted that temperature jump and slip velocity problems arise even at the Navier Stokes level and are not reduced to a purely Knudsen layer description problem even if a consistent Knudsen layer description can give a correction on the results provided by the Navier Stokes level calculations [14]. Then, $f^{-}$is written

$$
f^{-}=f_{M}[1+\varphi]
$$

where $f_{M}\left(V^{\prime}\right)=\frac{n}{\left(C_{0} \sqrt{\pi}\right)^{3}} e^{-\frac{V^{\prime 2}}{C_{0}^{2}}}$ is the Maxwellian at temperature $T_{0}$ of the gas and $\varphi\left(V^{\prime}\right)$ is a deviation from the Maxwellian, written in its complete Enskog approximation form for a simple gas $[9]$

$$
\varphi\left(V^{\prime}\right)=\frac{m}{k T_{0}}\left[\frac{\lambda_{c}}{n k T_{0}} \nabla T . V^{\prime}\left(1-\frac{2}{5 C_{0}^{2}} V^{\prime 2}\right)+\frac{\mu}{n k T_{0}}\left(V_{i}^{\prime} V_{j}^{\prime}-\frac{1}{3} V^{\prime 2} \mathbb{I}\right): \frac{\partial U_{i}}{\partial X_{j}}\right] .
$$

II denotes the second order identity tensor, ":" denotes the double contraction of the tensorial product, $V_{i}^{\prime} V_{j}^{\prime}$ and $\frac{\partial U_{i}}{\partial X_{j}}$ are the usual second order tensors. $\mu$ is the shear viscosity. Let us note that in expression (10), the chapman Enskog distribution is written at the wall, thus the sign of the velocity gradient is inverted, according to the theoretical kinetic theory definition of the gas mean velocity at the boundary (see the definition of the gas mean velocity at the wall in section IV) [2].

In regard to relations (7) and (9) let us note

$$
Q_{0}=Q_{0 M}+Q_{0 \varphi}
$$


where $Q_{0 M}$ denotes the contribution of $f_{M}\left(V^{\prime}\right)$ and $Q_{0 \varphi}$ denotes the contribution of $\varphi\left(V^{\prime}\right)$ to $Q_{0}$. The calculations of $Q_{0 M}$ and $Q_{0 \varphi}$, summarized in the Appendix, yield:

$$
Q_{0 M}=\frac{m n C_{0}}{4 \sqrt{\pi}} \sigma_{0}\left(C_{0}^{2}-C_{w}^{2}\right)-\frac{m n C_{0}}{4 \sqrt{\pi}}\left(\alpha_{y} U_{y}^{2}+\alpha_{z} U_{z}^{2}\right)
$$

and

$$
\begin{array}{r}
Q_{0 \varphi}=\frac{\sigma_{1}}{20} \frac{m \lambda_{c}}{k T_{0}} C_{0}^{2} \frac{\partial T}{\partial x}-\frac{\mu m C_{0}}{12 k T_{0} \sqrt{\pi}}\left(\sigma_{0} C_{w}^{2}+\alpha_{y} U_{y}^{2}+\alpha_{z} U_{z}^{2}\right)\left[3 \frac{\partial U_{x}}{\partial x}-\nabla . U\right]+ \\
\frac{\mu m C_{0}^{3}}{4 k T_{0} \sqrt{\pi}}\left[3 \alpha_{x} \frac{\partial U_{x}}{\partial x}+\frac{\alpha_{y}-\alpha_{z}}{2} \frac{\partial U_{y}}{\partial y}+\frac{\alpha_{z}-\alpha_{y}}{2} \frac{\partial U_{z}}{\partial z}\right]
\end{array}
$$

with

$$
\sigma_{1}=3 \alpha_{x}+\alpha_{y}+\alpha_{z}
$$

\section{A. A complete thermal boundary condition.}

The complete expression of the heat flux $Q_{0}$ is obtained by adding expressions (12) and (13). Then, applying condition (2) we deduce the expression of $C_{0}^{2}-C_{w}^{2}$. Finally, from $C_{0}^{2}-C_{w}^{2}=\frac{2 k}{m}\left(T_{0}-T_{w}\right)$, we obtain:

$$
\begin{array}{r}
\left(T_{0}-T_{w}\right)-\frac{\mu}{n k}\left(\frac{\partial U_{x}}{\partial x}-\frac{1}{3} \nabla \cdot U\right)\left[\frac{T_{w}}{T_{0}}+\frac{1}{\sigma_{0} C_{0}^{2}}\left(\alpha_{y} U_{y}^{2}+\alpha_{z} U_{z}^{2}\right)\right]=\frac{\lambda_{c} \sqrt{\pi}}{n k C_{0}}\left(\frac{2-\frac{\sigma_{1}}{10}}{\sigma_{0}}\right) \frac{\partial T}{\partial x} \\
-\frac{\mu}{n k}\left[\frac{3 \alpha_{x}}{\sigma_{0}} \frac{\partial U_{x}}{\partial x}+\frac{\alpha_{y}-\alpha_{z}}{2 \sigma_{0}} \frac{\partial U_{y}}{\partial y}+\frac{\alpha_{z}-\alpha_{y}}{2 \sigma_{0}} \frac{\partial U_{z}}{\partial z}\right]+\frac{T_{0}}{\sigma_{0} C_{0}^{2}}\left(\alpha_{y} U_{y}^{2}+\alpha_{z} U_{z}^{2}\right) .
\end{array}
$$

In equation (15) each gradient is taken at the position $x=0$, i.e. its value at the boundary obtained from the continuum equations.

The approach used above to derive the thermal boundary condition may be commented in the following way: first, as a consequence of the weak influence of the temperature and density variation in the Knudsen layer $[6,25,26]$ the double formulation of the heat flux may be considered as meaning that the heat flux is conserved through this layer. This assumption is not surprising at all, since it is usually considered as a basic property of the Knudsen layer [6]. Secondly, it is admitted that the distribution function of the incoming particles is not significantly changed by the gas/gas collisions occurring in the Knudsen layer. Therefore, 
we use a Chapman Enskog approximation for the incoming particle distribution function considered as frozen along the Knudsen layer. So we can write relation (15), confusing the value of the normal temperature gradient at the Knudsen layer inlet with its value at the wall. Although used by several authors $[2,13,16]$ this latter assumption is not completely founded in some situations, especially when the flow is not sufficiently rarefied or when the temperature jump is of the same order as the temperature itself [13].

\section{B. General comment on the thermal boundary condition (15)}

Relation (15) is a three-dimensional thermal boundary condition based on the complete Chapman-Enskog approximation for the incoming distribution function. It appears that this relation is structured in the same way as the continuum energy equation. The second term on the left-hand-side and the second term on the right-hand-side including the velocity gradients may be associated to the shear work at the solid boundary due to the gas motion. These two terms involve notably the gas compressibility and the surface anisotropy effects. The first term on the right-hand-side is due to the conduction heat flux associated to the normal temperature gradient. Accordingly, relation (15) shows a contribution of local viscous heating to the temperature jump through the velocity gradients. Therefore, the temperature jump is no longer supported by the temperature gradient only. In any case, these terms involving velocity gradients should receive attention in the slip flow regime and especially in the flows interacting with an anisotropic surface. On both sides of formula (15), the last terms in which the square of the slip velocity component appears, may be associated to the macroscopic mechanical energy lost into heat energy due the wall interaction. However, the slip velocity $\left(U_{y}\right.$ and $U_{z}$ at $x=0$ ) is generally negligible compared to the thermal velocity $C_{0}$. This is especially obvious in microflows because the Mach number is small and thus the maximal streamwise is itself small compared to the thermal velocity. Furthermore, this is also admitted by various authors (Scott, Gupta, Shidlovsky, Patterson, et al ) in the spatial research field [27]. Therefore the terms including the slip velocities $\left(\frac{U_{y}^{2}}{C_{0}^{2}}\right.$ and $\left.\frac{U_{z}^{2}}{C_{0}^{2}}\right)$ will be 
neglected in expression (15) and the temperature jump relation is written in the following form:

$$
\begin{aligned}
\left(T_{0}-T_{w}\right)=\frac{\lambda_{c} \sqrt{\pi}}{n k C_{0}}\left(\frac{2-\frac{\sigma_{1}}{10}}{\sigma_{0}}\right) \frac{\partial T}{\partial x}-\frac{\mu}{n k}\left(\frac{3 \alpha_{x}}{\sigma_{0}}-\right. & \left.\frac{2 T_{w}}{3 T_{0}}\right) \frac{\partial U_{x}}{\partial x}-\frac{\mu T_{w}}{3 n k T_{0}}\left(\frac{\partial U_{y}}{\partial y}+\frac{\partial U_{z}}{\partial z}\right) \\
& -\frac{\mu}{n k}\left[\frac{\alpha_{y}-\alpha_{z}}{2 \sigma_{0}} \frac{\partial U_{y}}{\partial y}+\frac{\alpha_{z}-\alpha_{y}}{2 \sigma_{0}} \frac{\partial U_{z}}{\partial z}\right]
\end{aligned}
$$

The coefficient $\left(\frac{2-\frac{\sigma_{1}}{10}}{\frac{\sigma_{0}}{2}}\right)$ in front of the temperature gradient term substitutes the usual coefficient $\left(\frac{2-\sigma_{T}}{\sigma_{T}}\right)$ which appeared in the previous temperature jump relations $[8,15,16]$ where $\sigma_{T}$ was the thermal accommodation coefficient. Let us point out that, when $f^{-}$ used with the scattering kernel (1) is assumed to be a nondrifting Maxwellian distribution function, then $\frac{\sigma_{0}}{2}$ equals the energy accommodation coefficient (see Appendix ??). It is to note that some authors $[6,28]$ had previously put forward corrections on the first coefficient $\left(\frac{2-\sigma_{T}}{\sigma_{T}}\right)$ by means of approximated resolutions of the Boltzmann Equation in the Knudsen layer. So, Welander replaced coefficient $\left(\frac{2-\sigma_{T}}{\sigma_{T}}\right)$ by $\left(\frac{2-K \sigma_{T}}{\sigma_{T}}\right)$ where K, calculated numerically, is equal to 0.827. In our approach, the correction naturally lies on the interplay of the three momentum accommodation coefficients coming from the scattering kernel redefinition.

\section{Temperature jump on an isotropic surface: $\alpha_{y}=\alpha_{z}$}

The accommodation coefficients characterize both the gas and the surface. But some properties are only relevant to the surface. Therefore, the macroscopic isotropy of the surface implies that the corresponding scattering kernel must be invariant under rotation about the $x$ normal axis. Thus on such a surface, the two tangential momentum accommodation coefficients must be equal: $\alpha_{y}=\alpha_{z}$ [21]. Let us note that this surface property does not entail that the normal accommodation coefficient $\alpha_{x}$ should be equal to $\alpha_{y}$ or $\alpha_{z}$; thus the reflection process is not necessary globally isotropic. In this case, the temperature jump (16) is simplified:

$$
\begin{array}{r}
\left(T_{0}-T_{w}\right)=\frac{\lambda_{c} \sqrt{\pi}}{n k C_{0}}\left(\frac{\left.2-\frac{3 \alpha_{x}+2 \alpha_{y}}{10}\right)}{\alpha_{x}+\alpha_{y}}\right) \frac{\partial T}{\partial x}-\frac{\mu}{n k}\left(\frac{3 \alpha_{x}}{\alpha_{x}+\alpha_{y}}-\frac{2 T_{w}}{3 T_{0}}\right) \frac{\partial U_{x}}{\partial x}- \\
\frac{\mu T_{w}}{3 n k T_{0}}\left(\frac{\partial U_{y}}{\partial y}+\frac{\partial U_{z}}{\partial z}\right)
\end{array}
$$


where, according to previous remarks, the terms in $\frac{U_{y}^{2}}{C_{0}^{2}}$ have been cancelled.

In the temperature jump relation (17) the novel two terms at the end in the left-handside represent the contribution of the gas friction on the wall (including compressibility effects) to the temperature jump at the wall. The weight of these terms depends on the flow configuration and on the temperature ratio $\frac{T_{w}}{T_{0}}$.

\section{Dimensional analysis in particular flow configurations}

\section{One-dimensional flow normal to the wall.}

If the flow streamwise is normal to the wall and the surface is supposed to be an isotropic plane surface at uniform temperature, then the tangential gradients vanish in relation (16) ( or in relation (17)).

We use the following dimensionless quantities,

$$
T^{*}=\frac{T}{T_{w}}, \quad U_{x}^{*}=\frac{U_{x}}{U_{e}}, \quad x^{*}=\frac{x}{h}
$$

where $U_{e}$ and $T_{e}$ are respectively a constant speed and a constant temperature characterizing the flow far from the wall. The characteristic length $h$ is chosen equal to the characteristic length of the Knudsen layer close to the wall. Note

$$
K_{T}=\frac{2-\frac{\sigma_{1}}{10}}{\frac{\sigma_{0}}{2}}, \quad K_{x}=\frac{3 \alpha_{x}}{\sigma_{0}}, \quad \alpha_{y}=\alpha_{z} .
$$

The simplified temperature jump takes the form

$$
\frac{T_{0}-T_{w}}{T_{w}}=\frac{\lambda_{c}}{n k h} \sqrt{\frac{\pi m}{2 k T_{0}}}\left\{\frac{K_{T}}{2} \frac{\partial T^{*}}{\partial x^{*}}-\frac{k \mu}{m \lambda_{m}} U_{e} \sqrt{\frac{2 m}{\pi k T_{0}}}\left(K_{x} \frac{T_{0}}{T_{w}}-\frac{2}{3}\right) \frac{\partial U_{x}^{*}}{\partial x^{*}}\right\}
$$

Now using the following relation between the thermal conductivity and the shear viscosity, $\frac{k \mu}{m \lambda_{c}}=\frac{4}{15}[9,29]$, which requires no particular choice of interaction model apart from the basic Chapman-Enskog assumptions. Then defining the mean free path by $\lambda_{m}=\frac{\mu}{m n} \sqrt{\frac{\pi m}{2 k T_{0}}}$ as proposed by Cercignani [14], the dimensionless temperature jump relation may be rewritten in the following form:

$$
T_{0}^{*}-1=K_{n}\left\{K_{T} \frac{15}{8} \frac{\partial T^{*}}{\partial x^{*}}-\sqrt{\frac{6}{\pi}} \Re\left(K_{x} T_{0}^{*}-\frac{2}{3}\right) \frac{\partial U_{x}^{*}}{\partial x^{*}}\right\}
$$


where $K_{n}=\frac{\lambda_{m}}{h}$, the Knudsen number characterizing the Knudsen layer is close to 1 . The factor $\Re$, defined by $\Re^{2}=\frac{\frac{1}{2} m U_{e}^{2}}{\frac{3}{2} k T_{0}}$ may be written using $M a_{e}$ the Mach number far from the wall, $\Re=\frac{\sqrt{5}}{3} M a_{e} \sqrt{\frac{T_{e}}{T_{0}}}$.

Such a configuration, where the flow is normal to the surface, seems convenient for modelling the boundary conditions of unsteady flows on the nose thermal shield of spatial vehicles during atmospheric re-entries. In these conditions, the $\Re$ factor is frequently of the magnitude order of 1 , since it is usual to obtain $M a_{e}$ slightly smaller than 1 whereas $\sqrt{\frac{T_{e}}{T_{0}}}$ does not exceed 4 or 5 [30]. On the other hand, to compare the gradient orders of magnitude, it is convenient to introduce the following dimensionless quantities:

$$
\theta=\frac{T-T_{w}}{T_{i}-T_{w}}, \quad \omega=\frac{U_{x}}{U_{i}}
$$

where the ${ }_{i}$ subscript characterizes the state of the gas at the Knudsen layer inlet. Then

$$
\frac{\partial T^{*}}{\partial x^{*}}=\frac{T_{i}-T_{w}}{T_{w}} \frac{\partial \theta}{\partial x^{*}}, \quad \frac{\partial U_{x}^{*}}{\partial x^{*}}=\frac{U_{i}}{U_{e}} \frac{\partial \omega}{\partial x^{*}},
$$

Here $\frac{\partial \theta}{\partial x^{*}}$ and $\frac{\partial \omega}{\partial x^{*}}$ are clearly of zero order, whereas $\frac{U_{i}}{U_{e}}$ reflects the scale ratio between the Knudsen layer and the characteristic length of the flow (i.e. the Knudsen number $K_{n e}$ far from the wall). So it appears from (19) that the relative influence of the velocity term in relation (18) depends on the thermal jump magnitude: if it is of the same order as the wall temperature the velocity terms are negligible. On the contrary, for small temperature jumps the model predicts a local viscous heating effect on the temperature jump, and the sign of this contribution may be inverted according to the value of the gas-to-wall temperature ratio.

\section{Flow in boundary layer configurations}

Let us consider relation (16) in a two-dimensional flow (the $x$ axis is normal to the wall and the $z$ axis components vanish), and let us perform the dimensional analysis of the configurations corresponding to Enskog boundary layers or to flows in microchannels. We 
define the dimensionless quantities as follows:

$$
T^{*}=\frac{T}{T_{w}}, \quad U_{x}^{*}=\frac{U_{x}}{V_{n}}, \quad U_{y}^{*}=\frac{U_{y}}{U_{s}}, \quad y^{*}=\frac{y}{L}, \quad x^{*}=\frac{x}{h} .
$$

$L$ is the characteristic length of the wall, $h$ the boundary layer thickness or the channel height. $U_{e}$ and $V_{n}$ respectively refer to the tangential and normal velocity in the flow far from the wall. $U_{s}$ is a constant characteristic velocity slip of the gas at the wall.

Note $K_{y}=\frac{\alpha_{y}-\alpha_{z}}{2 \sigma_{0}}, \epsilon_{n}=\frac{V_{n}}{U_{e}}, \epsilon_{h}=\frac{h}{L}, \eta^{0}=\frac{U_{s}}{U_{e}}$; the bi-dimensional temperature jump can be written in the following dimensionless form :

$$
T_{0}^{*}-1=K_{n}\left\{K_{T} \frac{15}{8} \frac{\partial T^{*}}{\partial x^{*}}-\sqrt{\frac{6}{\pi}} \Re\left[\left(K_{x} T_{0}^{*}-\frac{2}{3}\right) \epsilon_{n} \frac{\partial U_{x}^{*}}{\partial x^{*}}-\left(K_{y} T_{0}^{*}+\frac{1}{3}\right) \eta^{0} \epsilon_{h} \frac{\partial U_{y}^{*}}{\partial y^{*}}\right]\right\} .
$$

where $\Re$ is defined exactly as it is in the normal flow configuration.

Considering this flow configuration it appears clearly that $\frac{h}{L} \approx \frac{V_{n}}{U_{e}}<<1$. Otherwise $\frac{U_{s}}{U_{e}}<1$ and then $\eta^{0} \epsilon_{h}<\epsilon_{n}<<1$. Here any star-marked quantity is of the order of 1 . Moreover in a classical boundary layer $\Re$ is usually of the same order as in the case of the flow normal to the wall previously analyzed, i.e. $\Re \approx 1$. But in the microflow fields, the Mach number is generally small compared to $1(\approx 0.1-0.2)$ and the ratio $\frac{T_{0}}{T_{e}}$ is not very different from 1 [31]. Consequently $\Re$ rarely exceeds 0.1 and the relative weight of the viscous terms on the temperature jump is generally very small; then their effects should be negligible in ordinary microflows.

In a simple configuration where the gas is assumed to be moving tangentially along the wall, only the velocity gradient along the $y$ axis subsists in relations (16) or (17). Then the viscous heating effects at the wall lie only on the fluid acceleration along the wall. In spite of its theoretical simplicity, this case is not realistic along an isotropic surface: because such a unidirectional flow field would exist only if the compressibility were neglected (as in Poiseuil flow), then $\nabla \cdot U=0$ and no velocity gradient would subsist in relation (17). But it is different when gradients coming from anisotropy are present as in relations (16) or (20): then even if the compressibility is negligible, unidirectional flows involving small viscous heating at the wall can appear. 


\section{ON THE SLIP VELOCITY}

In the same way as the gas temperature at $x=0$ was derived from the account of heat flux, the mean velocity of the gas at $x=0$ can be derived from the account of the mass flux. This mass flux account at the wall leads us to calculate the mean velocity using averages based on the particle distribution function as formulated in the kinetic theory. But as was pointed out by Maxwell [2], considering the momentum components, the macroscopic meaning of these averages calculated at the wall is reversed compared to the meaning taken in any point within the gas. So, it yields at $x=0$

$$
-n U=\int_{\Omega^{\prime}} \xi^{\prime} f^{-} d_{\xi^{\prime}}+\int_{\Omega} \xi f^{+} d_{\xi} .
$$

Project relation (21) on the $x$ axis. Using relation (5) between $f^{+}$and $f^{-}$and the normalization condition on the scattering kernel, it is found $U_{x}=0$, due to the surface impermeability.

\section{A. Calculation of the slip velocity}

Project relation (21) on the $y$ axis. Using relation (5) between $f^{+}$and $f^{-}$, it is obtained at $x=0$,

$$
n U_{y}=\int_{\Omega^{\prime}} \xi_{x}^{\prime}\left[\int_{\Omega} \frac{\xi_{y}}{\xi_{x}} B\left(\xi^{\prime}, \xi\right) d_{\xi}\right] f^{-} d_{\xi^{\prime}}-\int_{\Omega^{\prime}} \xi_{y}^{\prime} f^{-} d_{\xi^{\prime}} .
$$

The integral $\int_{\Omega} \frac{\xi_{y}}{\xi_{x}} B\left(\xi^{\prime}, \xi\right) d_{\xi}$ may be calculated separately with $B\left(\xi^{\prime}, \xi\right)$ given in relation (1). This calculation gives

$$
\int_{\Omega} \frac{\xi_{y}}{\xi_{x}} B\left(\xi^{\prime}, \xi\right) d_{\xi}=\alpha_{x}\left(1-\alpha_{y}\right) \frac{\sqrt{\pi}}{C_{w}} \xi_{y}^{\prime}-\left(1-\alpha_{x}\right)\left(1-\alpha_{y}\right) \frac{\xi_{y}^{\prime}}{\xi_{x}^{\prime}} .
$$

Therefore,

$$
n U_{y}=\alpha_{x}\left(1-\alpha_{y}\right) \frac{\sqrt{\pi}}{C_{w}} \int_{\Omega^{\prime}} \xi_{x}^{\prime} \xi_{y}^{\prime} f^{-} d_{\xi^{\prime}}-\left[1+\left(1-\alpha_{x}\right)\left(1-\alpha_{y}\right)\right] \int_{\Omega^{\prime}} \xi_{y}^{\prime} f^{-} d_{\xi^{\prime}} .
$$

Now, choosing the same incoming distribution function as for the thermal jump calculation (relation (9)) and using the change $\xi^{\prime}=V^{\prime}+U$, relation (22) splits into

$$
\begin{gathered}
n U_{y}=\alpha_{x}\left(1-\alpha_{y}\right) \frac{\sqrt{\pi}}{C_{w}}\left[U_{y} J_{0 x}+U_{y} J_{2}+J_{4}\right]- \\
\left(1+\left(1-\alpha_{x}\right)\left(1-\alpha_{y}\right)\right)\left[U_{y} J_{0}+U_{y} J_{1}+J_{3}\right],
\end{gathered}
$$


where are denoted:

$$
\begin{array}{rr}
J_{0}=\int_{\Omega^{\prime}} f_{M} d_{V^{\prime}} & J_{0 x}=\int_{\Omega^{\prime}} V_{x}^{\prime} f_{M} d_{V^{\prime}} \\
J_{1}=\int_{\Omega^{\prime}} \varphi f_{M} d_{V^{\prime}} & J_{2}=\int_{\Omega^{\prime}} V_{x}^{\prime} \varphi f_{M} d_{V^{\prime}} \\
J_{3}=\int_{\Omega^{\prime}} V_{y}^{\prime} \varphi f_{M} d_{V^{\prime}} & J_{4}=\int_{\Omega^{\prime}} V_{x}^{\prime} V_{y}^{\prime} \varphi f_{M} d_{V^{\prime}} .
\end{array}
$$

$J_{0}$ and $J_{0 x}$ give easily $J_{0}=\frac{n}{2}$ and $J_{O x}=-\frac{n C_{0}}{2 \sqrt{\pi}}$. The calculations of $J_{1}, J_{2}, J_{3}$, and $J_{4}$, involve the complex expression of $\varphi$ given in relation (10). However these calculations are feasible and it is found

$$
\begin{array}{r}
J_{1}=-\frac{3}{4} \frac{\mu}{m T_{0} C_{0} \sqrt{\pi}} \frac{\partial T}{\partial x} \quad J_{2}=-\frac{\mu}{m C_{0} \sqrt{\pi}}\left(\frac{\partial U_{x}}{\partial x}-\frac{1}{3} \nabla \cdot U\right) \\
J_{3}=-\frac{\mu}{m C_{0} \sqrt{\pi}}\left(\frac{\partial U_{x}}{\partial y}+\frac{\partial U_{y}}{\partial x}\right) \quad J_{4}=\frac{3}{8} \frac{\mu C_{0}}{m T_{0} \sqrt{\pi}} \frac{\partial T}{\partial y}+\frac{\mu}{2 m}\left(\frac{\partial U_{x}}{\partial y}+\frac{\partial U_{y}}{\partial x}\right) .
\end{array}
$$

Relation (23) can be arranged in the form

$$
\begin{array}{r}
U_{y}\left(4-\gamma+\beta \frac{C_{0}}{C_{w}}\right)-\beta \frac{U_{y}}{C_{0}} \frac{2 C_{0} \sqrt{\pi}}{n C_{w}} J_{2}+(2-\gamma) \frac{U_{y}}{C_{0}} \frac{2 C_{0}}{n} J_{1}= \\
-(2-\gamma) \frac{2}{n} J_{3}+\beta \frac{2 \sqrt{\pi}}{n C_{w}} J_{4}
\end{array}
$$

with

$$
\beta=\alpha_{x}\left(1-\alpha_{y}\right), \quad \gamma=\alpha_{y}+\alpha_{x}\left(1-\alpha_{y}\right)
$$

Finally, from (25) and (24), the complete boundary condition on the $y$ component of the tangential mean velocity is written in the following form

$$
\begin{array}{r}
U_{y}\left(4-\gamma+\beta \sqrt{\frac{T_{0}}{T_{w}}}\right)+\beta \frac{U_{y}}{C_{0}^{2}} \frac{2 \mu}{m n} \sqrt{\frac{T_{0}}{T_{w}}}\left(\frac{\partial U_{x}}{\partial x}-\frac{1}{3} \nabla \cdot U\right)- \\
(2-\gamma) \frac{U_{y}}{C_{0}} \frac{3 \mu}{2 m n T_{0} \sqrt{\pi}} \frac{\partial T}{\partial x}= \\
\frac{2 \mu}{m n C_{0} \sqrt{\pi}}\left(2-\gamma+\beta \frac{\pi}{2} \sqrt{\frac{T_{0}}{T_{w}}}\right)\left(\frac{\partial U_{x}}{\partial y}+\frac{\partial U_{y}}{\partial x}\right)+\beta \frac{3 \mu}{4 m n T_{0}} \sqrt{\frac{T_{0}}{T_{w}}} \frac{\partial T}{\partial y}
\end{array}
$$

As for the thermal boundary condition, the effects of using an Enskog distribution function for $f^{-}$will be commented in the conclusion. However, let us point out that through relation (21) we used the basic definition of the mean velocity in kinetic theory, instead of the usual 
rough momentum balancing generally used in slip velocity derivation $[2,8,9]$. Thus the new approach appears as a direct calculation of the slip velocity, consistent with the kinetic theory formulation from the momentum components and accounting for the singularity of this formulation at the wall.

\section{B. Simplified expression of the slip velocity}

Relation (26) is a complete boundary condition on the tangential velocity for the continuum equations. However, in this relation, the term involving the velocity gradients on the left-hand-side and the term involving the velocity gradients on the right-hand-side are in a magnitude order of the ratio $\frac{U_{y}}{C_{0}}$. It is the same for the terms involving the temperature gradients. As mentioned before in the discussion about the thermal boundary condition, the slip velocity of the gas at the wall is negligible compared to the thermal velocity (at $x=0, U_{y}^{2}<<C_{0}^{2}$ ), thus the last two terms on the left hand side of relation (26) can be neglected: the dimensional analysis (section IV D) would confirm this choice with some restrictions for the normal temperature gradient term if a strong heat flux exists across the wall. Then the slip velocity component on the $y$ axis is written

$$
\begin{array}{r}
\left(4-\gamma+\beta \sqrt{\frac{T_{0}}{T_{w}}}\right) U_{y}= \\
\frac{2 \mu}{m n C_{0} \sqrt{\pi}}\left(2-\gamma+\beta \frac{\pi}{2} \sqrt{\frac{T_{0}}{T_{w}}}\right)\left(\frac{\partial U_{x}}{\partial y}+\frac{\partial U_{y}}{\partial x}\right)+\beta \frac{3 \mu}{4 m n T_{0}} \sqrt{\frac{T_{0}}{T_{w}}} \frac{\partial T}{\partial y} .
\end{array}
$$

Naturally, the corresponding expression of the slip velocity on the $z$ axis might be written in the same way transposing $y$ and $z$ notations. In relation (27) the tangential gradient of the normal mean velocity component appears: it was generally missing in previous approach formulae [2] and recently pointed out as necessary [32]. In the usual terms (thermal creep and $U_{y}$ normal gradient) the slip coefficient and the thermal creep coefficient depend on the gas-to-wall temperature ratio $\frac{T_{0}}{T_{w}}$ and on the various momentum accommodation coefficients (notably on the normal accommodation coefficient). In the usual cases where the wall is an isotropic surface $\left(\alpha_{y}=\alpha_{z}\right)$, coefficients $\gamma$ and $\beta$ are the same in the expressions of the two components $U_{y}$ and $U_{z}$ of the slip velocity: thus $U_{y}$ and $U_{z}$ refer to a unique vectorial 
equation for the slip velocity along the surface plane. Therefore, in any case the present model is suitable to treat a three dimensional flow configuration.

When the reflecting surface is considered as an ideal specular reflecting surface $\left(\alpha_{x}=\right.$ $\alpha_{y}=\alpha_{z}=0$ ), two significant results are predicted from relation (27), which cannot be derived from previous models. First, when all the accommodation coefficients vanish in relation (27), the "thermal creep" vanishes too. This confirms theoretically the "thermal transpiration" qualitative explanation provided by Maxwell and Reynolds and adopted so far, considering the "thermal creep" as resulting from a gas/surface interaction effect [2, 33]. Consequently, the thermal creep should vanish when the boundary condition becomes pure geometrical condition, as it is provided by a perfect specular reflecting surface [14] where the gas does not exert any stress. Contrary to relation (27) the classical slip models predict in this case a thermal creep effect remaining anomalously present. Second, when all the accommodation coefficients vanish, the present formula (27), which is a direct calculation of the slip, leads to a correct qualitative response,

$$
U_{y}=\frac{\mu}{m n C_{0} \sqrt{\pi}}\left(\frac{\partial U_{x}}{\partial y}+\frac{\partial U_{y}}{\partial x}\right) \simeq \frac{\lambda_{m}}{\mu} \tau
$$

where $\tau$ is the tangential shear stress at the wall. This result means that on a specular surface the slip velocity is practically the gas mean velocity at a distance equal to the mean free path from the wall, as phenomenologically predicted $[1,9,18]$. On the contrary, as soon as the Maxwell accommodation coefficient (usually associated to the tangential accommodation coefficient) vanishes, the Maxwell slip coefficient becomes infinite, and so the velocity slip remains unknown whatever the stress behavior at the wall.

\section{Comparison with experimental measurements of slip coefficient}

Before comparing our slip velocity results with the Maxwell approach and with experimental results, a preliminary remark is needed. In expression (10), according to ChapmanEnskog assumption (see pages $173-175$ of reference [9]), the viscosity coefficient derives from the well-known inverse fifth power law interaction potential (Maxwellian molecules): 
such a model does not lead exactly to the usual mean free path expressed by $\lambda_{m}=\frac{\mu}{m n} \sqrt{\frac{\pi m}{2 k T_{0}}}$, which corresponds to the Maxwell phenomenological assumption (see pages 231, 240 and the Appendix of reference [2]) and which remains close to the hard sphere model mean free path. Therefore, it is necessary to correct the viscosity coefficient appearing in the present slip velocity calculations using a factor $\frac{3 \pi}{2}$ in order to be consistent with the formal $\lambda_{m}$ expression. This is obtained by fitting both the Maxwell slip velocity and the present slip expression in the case of diffuse reflection where the Maxwell expression is known to give satisfactory results[14]. Then, from the slip velocity relation (27) a slip length can be deduced

$$
\zeta_{0}=\frac{3 \mu \sqrt{\pi}}{m n C_{0}} \frac{\left(2-\gamma+\beta \frac{\pi}{2} \sqrt{\frac{T_{0}}{T_{w}}}\right)}{\left(4-\gamma+\beta \sqrt{\frac{T_{0}}{T_{w}}}\right)} .
$$

The corresponding slip coefficient, $\sigma_{v}$, derived using relation $\zeta_{0}=\sigma_{v} \lambda_{m}$, reads

$$
\sigma_{v}=3 \frac{\left(2-\gamma+\beta \frac{\pi}{2} \sqrt{\frac{T_{0}}{T_{w}}}\right)}{\left(4-\gamma+\beta \sqrt{\frac{T_{0}}{T_{w}}}\right)}
$$

From the interplay of the normal and the tangential accommodation coefficients one can see that the slip length from relation (29) depends slightly on the accommodation coefficients and remains close to $\lambda_{m}$. Precisely, the present relation (30) predicts that, when the temperature jump is negligible, $\sigma_{v}$ ranges from 1 to 1.93 when the gaz/surface properties vary through the variation of the accommodation coefficient values (Table II). On the contrary, the Maxwell expression of the slip length $\frac{2-\alpha_{y}}{\alpha_{y}} \lambda_{m}$ increases strongly for vanishing values of the Maxwell accommodation coefficient. The slip coefficient prediction based on the Maxwell type of boundary condition should be called into question as soon as the physical conditions move away from the perfect accommodation and especially for small values of the accommodation coefficients (see table I).

Table III presents results of slip coefficient measurements carried out by Porodnov et.al. [34] for various gases on various surfaces. These are direct measurements of the slip coefficient without any arbitrary fixed value of the accommodation coefficients. Table III shows that when the couple gaz/surface properties vary the measured slip coefficient values lie between 
1 and 2. Consequently, taking into account the correction on the viscosity theoretical calculation, and so on the mean free path definition at the wall, as pointed out above, we observe that the slip coefficients predicted by relation (30) are in agreement with the experimental values of reference [34].

\section{Dimensional analysis in boundary layer configurations}

In a boundary layer or in a microflow configuration, we analyze equation (27) in the same way which led to relation (20) Using the same parameters as those defined in section (III C 2), supplemented with $\eta_{y}=\frac{U_{y}}{U_{e}}$, we obtain :

$$
\left(4-\gamma+\beta \sqrt{T_{0}^{*}}\right) \eta_{y}=\frac{3}{2} K_{n}\left(2-\gamma+\beta \frac{\pi}{2} \sqrt{T_{0}^{*}}\right)\left(\eta^{0} \frac{\partial U_{y}^{*}}{\partial x^{*}}+\epsilon_{h} \epsilon_{n} \frac{\partial U_{x}^{*}}{\partial y^{*}}\right)+\beta \epsilon_{h} \frac{\sqrt{6 \pi}}{8 \Re} \frac{\partial T^{*}}{\partial y^{*}}
$$

As in equation (20) the star-marked quantities are all of the order of 1 . Moreover $\eta^{0}$ is usually found in the [0.1-0.2] range. Therefore, on the basis of the previous comments, it appears that the thermal creep has generally a weight $\left(\frac{\epsilon_{h}}{\Re}\right)$ of the same order as the weight $\eta^{0}$ of the normal gradient of the tangential velocity. In usual microflow domains, the negligible effect of the tangential gradient of the normal velocity component seems confirmed. Nevertheless, it is to note that the above analysis is carried out neither for complex geometries involving strong surface curvature nor for particular motions of the wall. Such conditions have recently been mentioned as enhancing the effect of the missing terms in the usual slip formulae [32].

\section{CONCLUDING REMARKS}

Slip and jump boundary conditions at the wall were obtained analytically through a consistent approach based upon the kinetic theory. An anisotropic scattering kernel, previously derived, was employed to express the reflection law. The complete Chapman-Enskog distribution function involving the velocity gradients was used for the incoming particles. Two main aspects of the results may be pointed out. First, supplementary terms appear in the expressions of the boundary condition. In the temperature jump some terms involving various 
velocity gradients are present besides the normal temperature gradient term: these kinetic terms reflect the contribution to the temperature jump, provided by the gas friction against the solid surface; these viscous terms may account for the special behavior of the anisotropic reflecting surfaces. In the slip velocity the thermal creep is supplemented with new crossed gradients of the tangential velocity components, which some authors recently pointed out as missing in usual formulae. Second, in the usual terms the surface anisotropy can also be acting; thus the usual coefficients involved in the previous approaches are modified, including now three accommodation coefficients. Moreover, in the velocity slip coefficient, the wall-to-gas temperature ratio is also present.

As a result, the boundary conditions appear as a structured set of equation in which the coupling between the various gradients appears naturally. The coupling coefficient are linear combinations of the accommodations coefficient of the three momentum components. From a quantitative point of view, using dimensional analysis of the various geometrical configurations showed that some of the new terms are of the same magnitude order as the usual terms, showing the pertinence of the present approach. Finally the use of an incoming Chapman-Enskog distribution function requires a last comment: distribution functions involving the gas motion have rarely been employed so far to treat the thermal boundary conditions: in the eighties Scott and Gupta [16] used a Chapman-Enskog distribution to describe the gas behavior near the wall, but in a very different conceptual frame. Their approach was based on the gas property balances between the inlet of the Knudsen layer and the area in contact with the wall. Thus the temperature jump and the slip velocity appeared exclusively as a variation of the gas property across this layer and the wall itself was disregarded. The present approach is focused on the contact of the gas with the wall, and the influence of the Knudsen layer itself has not been realistically accounted for. The variation of the gradient through the Knudsen layer may be significant when a strong temperature difference occurs between the gas and the wall. But, in any case the present approach allows us to show the emergence of new phenomena in boundary conditions. A first comparison of the slip coefficient with experimental values partially validates the model and shows its 
usefulness. A complete exploitation needs theoretical and experimental investigations on the accommodation coefficient data.

\section{APPENDIX A: NOTICE ON THE DETAILED CALCULATIONS}

Let us write simply the scattering kernel $B\left(\xi^{\prime}, \xi\right)$ of relation (1) in the form

$$
B\left(\xi^{\prime}, \xi\right)=P_{x} P_{y} P_{z}
$$

where $P_{x}, P_{y}$, and $P_{z}$ correspond respectively to the three factors appearing in relation (1). These three factors $P_{x}, P_{y}$, and $P_{z}$ all satisfy

$$
\int_{0}^{+\infty} P_{x} d_{\xi_{x}}=\int_{-\infty}^{+\infty} P_{y} d_{\xi_{y}}=\int_{-\infty}^{+\infty} P_{z} d_{\xi_{z}}=1
$$

\section{On the calculation of $\int_{\Omega} V^{2} B\left(\xi^{\prime}, \xi\right) d_{\xi}$}

At $x=0$, taking into account $U_{x}=0$ it can be written

$$
V^{2}=(\xi-U)^{2}=\xi^{2}-2 \xi_{y} U_{y}-2 \xi_{z} U_{z}+U^{2}
$$

then

$$
\int_{\Omega} V^{2} B\left(\xi^{\prime}, \xi\right) d_{\xi}=\int_{\Omega} \xi^{2} B\left(\xi^{\prime}, \xi\right) d_{\xi}-2 U_{y} \int_{\Omega} \xi_{y} B\left(\xi^{\prime}, \xi\right) d_{\xi}-2 U_{z} \int_{\Omega} \xi_{z} B\left(\xi^{\prime}, \xi\right) d_{\xi}+U^{2} .
$$

Each of the integrals appearing in the right-hand-side of this latter equation can be calculated using a standard table for related integrals. Finally, remembering that $\xi^{\prime}=V^{\prime}+U$ the result may be written

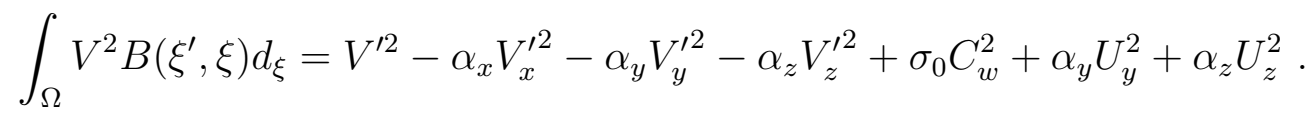

with $\sigma_{0}=\alpha_{x}+\frac{1}{2}\left(\alpha_{y}+\alpha_{z}\right)$

\section{On the calculation of $Q_{0 M}$}

$Q_{0 M}$ is written

$$
Q_{0 M}=\frac{1}{2} m \int_{\Omega^{\prime}}\left[\left(\alpha_{x} V_{x}^{\prime 2}+\alpha_{y} V_{y}^{\prime 2}+\alpha_{z} V_{z}^{\prime 2}\right)-\sigma_{0} C_{w}^{2}-\alpha_{y} U_{y}^{2}-\alpha_{z} U_{z}^{2}\right]\left|V_{x}^{\prime}\right| f_{M}\left(V^{\prime}\right) d_{V^{\prime}} .
$$


The integrals required for the $Q_{0 M}$ calculation are in the form $\int_{\Omega} V_{x}^{k} V_{y}^{l} V_{z}^{n} f_{M}(V) d_{V}$ where $k, l, n$ are integers. These integrals can be generated using a standard integral table of the exponential function, remembering that, according to our notation, $\left|V_{x}^{\prime}\right|=-V_{x}^{\prime}$ for $V_{x}^{\prime} \in \Omega^{\prime}$. The result reads

$$
Q_{0 M}=\frac{m n C_{0} \sigma_{0}}{4 \sqrt{\pi}}\left(C_{0}^{2}-C_{w}^{2}\right)-\frac{m n C_{0}}{4 \sqrt{\pi}}\left(\alpha_{y} U_{y}^{2}+\alpha_{z} U_{z}^{2}\right)
$$

\section{On the calculation of $Q_{0 \varphi}$}

$Q_{0 \varphi}$ is written,

$$
Q_{0 \varphi}=-\frac{1}{2} m \int_{\Omega^{\prime}}\left[\chi-\left(\alpha_{x} V_{x}^{\prime 2}+\alpha_{y}{V_{y}^{\prime 2}}^{2}+\alpha_{z} V_{z}^{\prime 2}\right)\right]\left|V_{x}^{\prime}\right| f_{M}\left(V^{\prime}\right) \varphi\left(V^{\prime}\right) d_{V^{\prime}}
$$

where $\chi=\sigma_{0} C_{w}^{2}+\alpha_{y} U_{y}^{2}+\alpha_{z} U_{z}^{2}$. Writing $\varphi\left(V^{\prime}\right)$ in the form

$$
\begin{gathered}
\varphi\left(V^{\prime}\right)=\frac{2 \lambda_{c}}{n k T C_{0}^{2}} \nabla T \cdot V^{\prime}-\frac{4 \lambda_{c}}{5 n k T C_{0}^{4}} V^{\prime 2} \nabla T . V^{\prime} \\
+\frac{2 \mu}{n k T C_{0}^{2}}\left(V_{i}^{\prime} V_{j}^{\prime}\right):\left(\frac{\partial U_{i}}{\partial X_{j}}\right)-\frac{2 \mu}{3 n k T C_{0}^{2}} V^{\prime 2} \nabla \cdot U
\end{gathered}
$$

the terms of $\varphi\left(V^{\prime}\right)$ which do not lead to vanishing integrals are the terms:

$$
\frac{\partial T}{\partial x} V_{x}^{\prime} ; \quad \frac{\partial T}{\partial x} V_{x}^{\prime} V^{\prime 2} ; \quad V_{x}^{\prime 2} \frac{\partial U_{x}}{\partial x} ; \quad V_{y}^{\prime 2} \frac{\partial U_{y}}{\partial y} ; \quad V_{z}^{\prime 2} \frac{\partial U_{z}}{\partial z} ; \text { and } \quad V^{\prime 2} \nabla \cdot U
$$

Then, using the relevant integral table of the form $\int_{\Omega} V_{x}^{k} V_{y}^{l} V_{z}^{n} f_{M}(V) d_{V}$, we find from this long calculation

$$
\begin{array}{r}
Q_{0 \varphi}=\frac{\sigma_{1}}{20} \frac{m \lambda_{c}}{k T_{0}} C_{0}^{2} \frac{\partial T}{\partial x}-\frac{\mu m C_{0}}{k T_{0} \sqrt{\pi}}\left[\frac{\partial U_{x}}{\partial x}\left(\frac{\chi}{6}-\frac{3 \alpha_{x}}{4} C_{0}^{2}\right)\right. \\
-\frac{\partial U_{y}}{\partial y}\left(\frac{\chi}{12}+\frac{\alpha_{y}-\alpha_{z}}{8} C_{0}^{2}\right) \\
\left.-\frac{\partial U_{z}}{\partial z}\left(\frac{\chi}{12}+\frac{\alpha_{z}-\alpha_{y}}{8} C_{0}^{2}\right)\right]
\end{array}
$$

$Q_{0 \varphi}$ can be easily rewritten in the form given in relation (13). 


\section{APPENDIX B: ENERGY ACCOMMODATION COEFFICIENT WHEN}

$$
f^{-}\left(V^{\prime}\right)=f_{M}\left(V^{\prime}\right), U=0
$$

The heat energy accommodation coefficient is defined by

$$
\frac{\Phi^{-}-\Phi^{+}}{\Phi^{-}-\Phi_{d}^{+}} .
$$

where $\Phi^{-}$is the incoming heat flux at the wall, $\Phi^{+}$is the reflected heat flux, and $\Phi_{d}^{+}$ is the reflected flux in the hypothetical situation of perfect accommodation to the wall. $\left(\Phi^{-}-\Phi^{+}\right)$is expressed exactly as in relation (4), (6) and (7). Then the particular case where $f^{-}\left(V^{\prime}\right)=f_{M}\left(V^{\prime}\right)$ yields

$$
\Phi^{-}-\Phi^{+}=\frac{m n C_{0} \sigma_{0}}{4 \sqrt{\pi}}\left(C_{0}^{2}-C_{w}^{2}\right)
$$

$\left(\Phi^{-}-\Phi_{d}^{+}\right)$can be calculated in the same way, substituting the scattering kernel $B\left(V^{\prime}, V\right)$ by the diffuse one, $B_{d}\left(V^{\prime}, V\right)=\frac{2}{C_{w}^{4} \pi} V_{x} e^{-\frac{V^{2}}{C_{w}^{2}}}$. It is found

$$
\Phi^{-}-\Phi_{d}^{+}=\frac{1}{2} m \int_{\Omega^{\prime}}\left|V_{x}^{\prime}\right| V^{\prime 2} f_{M}\left(V^{\prime}\right) d_{V^{\prime}}-m C_{w}^{2} \int_{\Omega^{\prime}}\left|V_{x}^{\prime}\right| f_{M}\left(V^{\prime}\right) d_{V^{\prime}}
$$

and finally,

$$
\Phi^{-}-\Phi_{d}^{+}=\frac{m n C_{0}}{2 \sqrt{\pi}}\left(C_{0}^{2}-C_{w}^{2}\right) .
$$

Consequently in this case the energy accommodation coefficient equals $\frac{\sigma_{0}}{2}$. 
[1] M. G. el hak, Mec. Ind. (Review) 2, 313 (2001).

[2] J. C. Maxwell, Philosophical Transactions of the royal society of London 170, 231 (1878).

[3] A. Kruger, Larmit, and M. D. Wit, in proceedings of the seventh International symposium on Rarefied Gas Dynamics (1970), pp. 1015-1022.

[4] A. Beskok and G. E. Karniadakis, AIAA Journal of Thermophysics and Heat Transfer 8, 647 (1994).

[5] C. M. Huang, R. V. Tompson, T. K. Ghosh, I. N. Ivchenko, and S. K. Loyalka, physics of fluids 11, 1662 (1999).

[6] P. Welander, Arkiv för Fysik 7, 507 (1954).

[7] Y. Sone, T. Ohwada, and K. Aoki, Physics of Fluids A: Fluid Dynamics 1, 363 (1989).

[8] E. H. Kennard, Kinetic Theory of Gases (McGraw-Hill, 1938).

[9] S. Chapman and T. Cowling, The Mathematical Theory of Non-Uniform Gases (Cambridge Mathematical Library, 1970), third edition ed.

[10] I. Ahmed and A. Beskok, Journal of Thermophysics and Heat Transfer 16, 161 (2002).

[11] Y. S. Ju, Journal of Heat Transfer 122, 817 (2000).

[12] N. G. Hadjiconstantinou, Journal of Heat Transfer 125, 944 (2003).

[13] M. N. Kogan, Prog. Aerospace Sci. 29, 271 (1992).

[14] C. Cercignani, Mathematical Methods in Kinetic Theory (plenum press new york, 1990), chap. 4, p. 98, 2nd ed.

[15] M. V. Smoluchowski, Wied. Ann. p. 101 (1899).

[16] R. N. Gupta, C. D. Scott, and J. N. Moss, AIAA Paper 84, 1732 (1984).

[17] H. Grad, in Communications on pure and applied mathematics (1949), vol. 2, pp. 331-407.

[18] S. A. Schaaf and P. L. Chambré, Flow of rarefied gases (Princeton, N.J., Princeton University Press, 1961).

[19] Y. Bayazitoglu and G. Tunc, Journal of Thermophysics and Heat Transfer 16, 472 (2002). 
[20] C. Cercignani, R. Illner, and M. Pulvirenti, The Mathematical Theory of Dilute Gases (Springer-Verlag, 1994).

[21] I. Kuscer, in International Symposium on Rarefied Gas Dynamics, Gottingen (1974), vol. E1, pp. $1-21$.

[22] S. Nocilla, Meccanica 2, 34 (1967).

[23] S. F. Shen, Entropie 18, 138 (1967).

[24] S. K. Dadzie and J. G. Meolans, Journal of Mathematical Physics 45, 1804 (2004).

[25] M. Larini, Ph.D. thesis, Université de Provence (1973).

[26] L. Aufrère and J. G. Méolans, C. R. Acad. Sci. Paris, Mécanique des fluides/fluids mechanics t.329 (2001).

[27] R. N. Gupta and C. D. Scott, AIAA Journal of Thermophysics and Heat Transfer 10, 190 (1996).

[28] M. Larini and R. Brun, International Journal of Heat and Mass Transfer 16, 2189 (1973).

[29] R. Brun, Transport et relaxation dans les ecoulements gazeux (Masson, 1986).

[30] J. J. Bertin, Hypersonic Aerothermodynamics, Education (AIAA, 1994).

[31] G. E. Karniadakis and A. Beskok, Micro Flows, Fundamentals and Simulation (Springer, 2001).

[32] D. A. Lockerby, J. M. Reese, D. R. Emerson, and R. W. Barber, Physical Review E 70, 017303 (2004).

[33] O. Reynolds, Philosophical Transactions of the royal society of London part 2 (1879).

[34] B. T. Porodonov, P. E. Suetin, S. F. Borisov, and V. D. Akinshin, Journal of Fluid Mechanics 64, 417 (1974). 


\section{TABLES}

\begin{tabular}{r|r|r|r|r|r|r|r|r}
\hline$\alpha_{y}$ & 1 & 0.8 & 0.7 & 0.6 & 0.5 & 0.4 & 0.2 & 0.1 \\
\hline$\sigma_{v}$ & 1 & 1.5 & 1.86 & 2.33 & 3 & 4 & 9 & 19
\end{tabular}

TABLE I: Maxwell slip coefficient calculated using relation $\frac{2-\alpha_{y}}{\alpha_{y}}$ for different values of $\alpha_{y}$.

\begin{tabular}{|c|c|c|c|c|c|c|c|c|c|}
\hline$\alpha_{x}$ & $\alpha_{y}=$ & 1 & 0.9 & 0.8 & 0.7 & 0.5 & 0.4 & 0.1 & 0 \\
\hline 1 & & 1 & 1.12 & 1.23 & 1.34 & 1.53 & 1.61 & 1.86 & 1.93 \\
\hline 0.9 & & 1 & 1.11 & 1.22 & 1.32 & 1.50 & 1.59 & 1.82 & 1.88 \\
\hline 0.7 & & 1 & 1.10 & 1.20 & 1.29 & 1.46 & 1.53 & 1.74 & 1.80 \\
\hline 0.4 & & 1 & 1.09 & 1.28 & 1.24 & 1.38 & 1.45 & 1.62 & 1.67 \\
\hline 0.1 & & 1 & 1.07 & 1.14 & 1.20 & 1.31 & 1.36 & 1.50 & 1.59 \\
\hline
\end{tabular}

TABLE II: Slip coefficient of relation (30) given for different values of $\alpha_{x}$ and $\alpha_{y}$.

\begin{tabular}{|c|c|c|c|c|c|c|c|}
\hline $\mathrm{He}$ & $\mathrm{Ne}$ & $\mathrm{Ar}$ & $\mathrm{Kr}$ & \multicolumn{2}{|c|}{$\mathrm{Xe} \quad \mathrm{H} 2$} & D2 & $\mathrm{CO} 2$ \\
\hline 1.24 & 1.25 & 1.31 & 1.23 & 1.33 & 1.22 & 1.27 & \\
\hline 1.49 & 1.56 & 1.46 & 1.39 & 1.38 & 1.37 & 1.41 & 1.24 \\
\hline 1.53 & 1.59 & 1.33 & 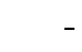 & - & - & 1.57 & - \\
\hline 1.02 & 1.71 & 1.35 & & - & 1.46 & 1.37 & \\
\hline
\end{tabular}

TABLE III: Experimental values of slip coefficients of various gases on various surfaces [34]. 